\title{
Solitary waves in fluid-filled elastic tubes: existence, persistence, and the role of axial displacement
}

\author{
Y.B. Fu ${ }^{1}$ and A. T. Il'ichev ${ }^{2}$ \\ ${ }^{1}$ Department of Mathematics, Keele University, ST5 5BG, U.K. \\ ${ }^{2}$ Steklov Mathematical Institute, Gubkina str. 8, 119991 Moscow, Russia
}

\begin{abstract}
We re-examine the problem of solitary wave propagation in a fluid-filled elastic membrane tube using a much simplified procedure. It is shown that there may exist four families of solitary waves with speeds close to those given by the linear dispersion relation, whether the fluid is initially stationary or not, and that it is not asymptotically consistent to neglect the axial displacement even in a long-wave approximation. It is also shown that the solitary wave solutions obtained by neglecting higher-order terms persist for the full system of equations in the sense that the full system has solutions of the solitary-wave type and each exact solution is uniformly approximated by the corresponding leading-order solution.
\end{abstract}

\section{Introduction}

Nonlinear wave propagation in arteries is a subject that has been much studied over the past three decades. As a very good approximation, the arterial blood flow can be modeled as an incompressible viscous or inviscid fluid flowing in a distensible elastic membrane tube. The linearized governing equations admit dispersive-wave solutions. Thus, when small but finite amplitude travelling waves are considered in the long wavelength limit, we expect to see the famous $\mathrm{KdV}$ equation, or $\mathrm{KdV}$-Burgers equation if viscous effects are taken into account, to emerge as the evolution equation for the wave amplitude. There now exists a good number of papers devoted to the derivation of the KdV or KdV-Burgers equation for arterial blood flows; see, for instance, Johnson (1971), Hashizume (1985), Cowley (1983), Yomosa (1987), Demiray (1996), Erbay et al. (1992), Demiray (1997), Demiray and Dost (1998), Antar and Demiray (1999) and the references therein. Various approximations have been adopted; some are ad hoc and some can be justified as being asymptotically self-consistent. In particular, we note that Cowley (1983) ignored the inertia of the membrane, Hashizume (1985) truncated his stress-strain relation for an unstressed membrane at the quadratic order, 
Yomosa (1987) approximated both the governing equation and the constitutive relation for the membrane, Demiray (1996) assumed that the axial displacement in the membrane can be neglected, whereas Demiray (1997) assumed that the axial displacement was so small that the governing equations can be linearized in terms of it. Most of these studies assume that the fluid is inviscid, and can be approximated by a one-dimensional model where the radial velocity and dependence on the radial variable can be neglected.

The present study is motivated by the results of Epstein and Johnston (2001, hereafter referred to as EJ) and those of $\mathrm{Fu}$ et al (2008, hereafter referred to as FPL), the latter authors examined the problem of localized bulging/necking in an inflated membrane tube with a view to model aneurysm formation. Whereas previous studies invariably used a multiple-scale perturbation procedure to derive the evolution equation, EJ noted that the problem has a variational formulation and, as a result, found two integrals (conservation laws) for the governing equations. They showed that solitary waves of finite amplitude can be determined exactly by a simple numerical procedure. Surprisingly, they did not specialize their analysis to the case when the speed in each case is close to the corresponding linear wave speed, thus failing to make a correct connection with the results obtained from a weakly nonlinear analysis. In fact, they seem to give the reader the impression that no solitary waves can propagate in a fluid-filled elastic membrane tube if the fluid is stationary prior to wave propagation. One of the aims of the present study is to show that when EJ's formulation is specialized to the case when the speed is close to a linear wave speed, it does yield a KdV equation, hence guaranteeing the existence of a family of solitary waves (parametrized by the wave speed). To this end, we use the formulation of FPL which can be viewed as an improved variation of EJ's original formulation. By deriving the KdV equation explicitly, first for the case when axial displacement is fully taken into account and then for the case when it is neglected, we shall also show that it is asymptotically inconsistent to neglect the axial displacement, even in the long-wave approximation. This has previously been noted by EJ by using a different argument.

The rest of this paper is organized into five sections as follows. In the next section, we quote the governing equations and the associated integrals, and rewrite them in a simplified form. The linear dispersion relation is noted down for later reference. We then apply, in Section 3, the procedure of FPL to derive a single amplitude equation of the form

$$
\left(\frac{d w}{d \bar{Z}}\right)^{2}=\omega\left(c, \lambda_{1 \infty}, \lambda_{2 \infty}\right) w^{2}+\gamma\left(c, \lambda_{1 \infty}, \lambda_{2 \infty}\right) w^{3}+O\left(w^{4}\right)
$$

for the radial displacement $w$, where $\bar{Z}=Z-\hat{c} t, Z$ is the axial coordinate, $\hat{c}$ is the wave speed with a non-dimensionalized value $c, \lambda_{1 \infty}$ and $\lambda_{2 \infty}$ are the two principal stretches in the tube prior to wave propagation, and $\omega\left(c, \lambda_{1 \infty}, \lambda_{2 \infty}\right)$ and $\gamma\left(c, \lambda_{1 \infty}, \lambda_{2 \infty}\right)$ each have an explicit expression for a general strain-energy function. For each fixed pair of $\left(\lambda_{1 \infty}, \lambda_{2 \infty}\right)$, $\omega\left(c, \lambda_{1 \infty}, \lambda_{2 \infty}\right)=0$ corresponds to the dispersion relation evaluated at zero wavenumber; whereas when $c=0$ and the fluid is stationary, $\omega\left(0, \lambda_{1 \infty}, \lambda_{2 \infty}\right)=0$ gives the bifurcation 
condition for the onset of localized bulging/necking, which connects with the analysis of FPL. In a small neighborhood of a linear speed, $c_{1}$ say, the full amplitude equation can then be approximated by

$$
\left(\frac{d w}{d \bar{Z}}\right)^{2}=\left(c-c_{1}\right) \frac{\partial}{\partial c} \omega\left(c_{1}, \lambda_{1 \infty}, \lambda_{2 \infty}\right) w^{2}+\gamma\left(c_{1}, \lambda_{1 \infty}, \lambda_{2 \infty}\right) w^{3},
$$

the derivative of which is then the KdV equation specialized to a travelling wave.

In Section 4, we compare (1.1) with its counterpart when the axial displacement is neglected. In the final section, we establish the persistence by proving that the solitary wave solution given by (1.2) is indeed a uniformly valid approximation of the exact solution governed by (1.1).

\section{Governing equations and the dispersion relation}

We consider propagation of nonlinear travelling waves in an infinite fluid-filled membrane tube of averaged radius $R$ and thickness $H$ in its unstressed configuration, assuming that the tube is made of an incompressible hyperelastic material and always maintains its axisymmetry. We assume that prior to wave propagation, the tube is already subjected to a finite deformation with principal stretches $\lambda_{1 \infty}$ and $\lambda_{2 \infty}$ in the circumferential and axial directions, respectively, and the fluid has constant speed $\hat{v}_{\mathrm{f} \infty}$ and exerts a constant pressure $H p_{\infty}$ on the tube wall (where $H$ is inserted to simplify the notation later). We use $Z$ to measure distance in the axial direction in the unstressed configuration, and $w(Z, t)$ and $u(Z, t)$ to denote the incremental displacement in the radial and axial directions, respectively. Thus, the position vector of a material particle in the tube in the current configuration has the form

$$
\boldsymbol{r}=r(Z, t) \boldsymbol{e}_{r}+z(Z, t) \boldsymbol{e}_{z}, \text { with } r=\lambda_{1 \infty} R+w, \quad z=\lambda_{2 \infty} Z+u,
$$

where $\boldsymbol{e}_{r}$ and $\boldsymbol{e}_{z}$ are unit vectors in the radial and axial directions, respectively.

Since the deformation is axially symmetric, the principal directions of stretch coincide with the lines of latitude (1-direction), the meridians (2-direction), and the normal to the deformed surface. Thus, the principal stretches are given by

$$
\lambda_{1}=\frac{r}{R}, \quad \lambda_{2}=\sqrt{z^{\prime 2}+r^{\prime 2}}, \quad \lambda_{3}=1 /\left(\lambda_{1} \lambda_{2}\right),
$$

where a prime denotes differentiation with respect to $Z$. The principal Cauchy stresses $\sigma_{1}, \sigma_{2}, \sigma_{3}$ in the deformed configuration for an incompressible material are given by

$$
\sigma_{i}=\lambda_{i} \hat{W}_{i}-\hat{p}, \quad i=1,2,3 \text { (no summation), }
$$

where $\hat{W}=\hat{W}\left(\lambda_{1}, \lambda_{2}, \lambda_{3}\right)$ is the strain-energy function, $\hat{W}_{i}=\partial \hat{W} / \partial \lambda_{i}$, and $\hat{p}$ is the pressure associated with the constraint of incompressibility; see, for instance, Ogden (1997). Utilizing the incompressibility constraint $\lambda_{1} \lambda_{2} \lambda_{3}=1$ and the membrane assumption $\sigma_{3}=0$ we find

$$
\sigma_{i}=\lambda_{i} W_{i}, \quad i=1,2 \text { (no summation), }
$$


where $W\left(\lambda_{1}, \lambda_{2}\right)=\hat{W}\left(\lambda_{1}, \lambda_{2}, \lambda_{1}^{-1} \lambda_{2}^{-1}\right)$ and $W_{1}=\partial W / \partial \lambda_{1}$ etc.

The governing equations for $u$ and $w$ can be obtained from exact field equations of general nonlinear shell theory, see, for instance, Budiansky (1968), but EJ gave a very readable selfcontained derivation. We quote their results and rewrite them in the form

$$
\begin{gathered}
{\left[R \sigma_{2} \frac{z^{\prime}}{\lambda_{2}^{2}}\right]^{\prime}-p r r^{\prime}=\rho R \ddot{u},} \\
{\left[R \sigma_{2} \frac{r^{\prime}}{\lambda_{2}^{2}}\right]^{\prime}-\frac{\sigma_{1}}{\lambda_{1}}+p r z^{\prime}=\rho R \ddot{w},}
\end{gathered}
$$

where $p$ is the fluid pressure divided by the wall thickness $H$ and a superimposed dot denote differentiation with respect time $t$. We note that these equations are valid even if $R$ is not a constant (e.g. if the initial configuration is a spherical shell in which case a prime in (2.5) and (2.6) denotes differentiation with respect to the arclength). However, in the rest of this paper we are only concerned with the case when $R$ is constant. Without loss of generality, we from now on take $R=1$.

With the fluid assumed to be inviscid and the fluid flow to be one-dimensional, EJ showed that for travelling waves with speed $\hat{c}$ the fluid equations can be integrated exactly, leading to the following simple expression for the scaled pressure:

$$
p=p_{\infty}+v_{\mathrm{f}}\left(1-\lambda_{1 \infty}^{4} / \lambda_{1}^{4}\right), \quad v_{\mathrm{f}} \equiv \frac{\rho_{\mathrm{f}}}{2 H}\left(\lambda_{2 \infty} \hat{c}-\hat{v}_{\mathrm{f} \infty}\right)^{2},
$$

where $\rho_{\mathrm{f}}$ is the density of the fluid.

Linearizing the governing equations (2.5) and (2.6) together with (2.7) in the neighborhood of

$$
\lambda_{1}=\lambda_{1 \infty}, \quad \lambda_{2}=\lambda_{2 \infty}, \quad u=w=0,
$$

and then looking for a travelling wave solution with wave number $\hat{k}$ and speed $\hat{c}$, we obtain the linear dispersion relation

$$
\begin{gathered}
\left(k^{2} m^{2}+2 m\right) c^{4}-4 m v_{\mathrm{f} \infty} c^{3}-\left(m \alpha_{0} k^{2}+m \gamma_{1} k^{2}-2 m v_{\mathrm{f} \infty}^{2}-m \beta_{0}+m \beta_{1}+2 \gamma_{1}\right) c^{2} \\
+4 v_{\mathrm{f} \infty} \gamma_{1} c-\left(\alpha_{1}-\beta_{0}\right)^{2}-2 v_{\mathrm{f} \infty}^{2} \gamma_{1}+k^{2} \alpha_{0} \gamma_{1}-\beta_{0} \gamma_{1}+\beta_{1} \gamma_{1}=0
\end{gathered}
$$

where

$$
\begin{gathered}
c=\frac{\lambda_{2 \infty}}{c_{0}} \hat{c}, \quad c_{0}=\sqrt{\frac{\mu h}{\rho_{\mathrm{f}} r_{0}}}, \quad m=\frac{\rho h}{\rho_{\mathrm{f}} r_{0}}, \quad v_{\mathrm{f} \infty}=\frac{\hat{v}_{\mathrm{f} \infty}}{c_{0}}, \quad k=\frac{\lambda_{1 \infty}}{\lambda_{2 \infty}} \hat{k}, \\
\mu \alpha_{0}=\lambda_{2 \infty} W_{2}^{(\infty)}, \quad \mu \alpha_{1}=\lambda_{1 \infty} \lambda_{2 \infty} W_{12}^{(\infty)}, \quad \mu \beta_{0}=\lambda_{1 \infty} W_{1}^{(\infty)} \\
\mu \beta_{1}=\lambda_{1 \infty}^{2} W_{11}^{(\infty)}, \quad \mu \gamma_{1}=\lambda_{2 \infty}^{2} W_{22}^{(\infty)}, \quad \mu \alpha_{2}=\frac{1}{2} \lambda_{1 \infty}^{2} \lambda_{2 \infty} W_{112}^{(\infty)}, \\
\mu \beta_{2}=\frac{1}{2} \lambda_{1 \infty}^{3} W_{111}^{(\infty)}, \quad \mu \gamma_{2}=\frac{1}{2} \lambda_{1 \infty} \lambda_{2 \infty}^{2} W_{122}^{(\infty)}, \quad \mu \gamma_{3}=\frac{1}{2} \lambda_{2 \infty}^{3} W_{222}^{(\infty)} .
\end{gathered}
$$

In the above expressions, $r_{0}\left(=\lambda_{1 \infty} R=\lambda_{1 \infty}\right)$ and $h$ are respectively the radius and thickness of the tube prior to wave propagation, $\mu$ is a typical material modulus, and the superscripts 
$(\infty)$ on the W's signify evaluation at $\lambda_{1}=\lambda_{1 \infty}, \lambda_{2}=\lambda_{2 \infty}$. In defining the constants in (2.9) and (2.10) we have followed the scheme of Demiray and Dost (1998).

When the inertia of the fluid is negligible, we may take the limit $\rho_{\mathrm{f}} \rightarrow 0$ in (2.8) to obtain

$$
\begin{aligned}
& k^{2}\left(m c^{2}\right)^{2}-\left(\alpha_{0} k^{2}+\gamma_{1} k^{2}-\beta_{0}+\beta_{1}\right)\left(m c^{2}\right) \\
& -\left(\alpha_{1}-\beta_{0}\right)^{2}+\alpha_{0} \gamma_{1} k^{2}+\left(\beta_{1}-\beta_{0}\right) \gamma_{1}=0
\end{aligned}
$$

where $m c^{2}=\lambda_{2 \infty}^{2} \rho \hat{c}^{2} / \mu$. To derive the conditions under which this equation has four real solutions for $c$, we first assume that the prestressed tube material is strongly elliptic, that is,

$$
\beta_{1}>0, \quad \gamma_{1}>0, \quad \beta_{1} \gamma_{1}-\alpha_{1}^{2}>0,
$$

see, e.g., Davies (1991). If the membrane tube were stress-free prior to wave propagation, we would have $\lambda_{1 \infty}=\lambda_{2 \infty}=1, \alpha_{0}=\beta_{0}=0$, and then the two solutions of (2.11) would be given by

$$
m c^{2}=\frac{1}{2 k^{2}}\left\{\gamma_{1} k^{2}+\beta_{1} \pm \sqrt{\left(\gamma_{1} k^{2}+\beta_{1}\right)^{2}-4 k^{2}\left(\beta_{1} \gamma_{1}-\alpha_{1}^{2}\right)}\right\} .
$$

These two solutions are both real and positive for all real $k$, yielding four branches for $c$ (two of them are minus the other two). A similar dispersion relation has previously been derived by Tait et al (1981) based on a slightly different model. Thus, by continuation the dispersion equation (2.8) has four real branches in a non-trivial domain in the space of $\left(\lambda_{1 \infty}-1, \lambda_{2 \infty}-1, \rho_{\mathrm{f}}, \hat{v}_{\mathrm{f} \infty}\right)$ that includes the origin. We note that with a non-zero $\hat{v}_{\mathrm{f} \infty}$ breaking the symmetry, these four branches all have different characters, unlike the case of $\hat{v}_{\mathrm{f} \infty}=0$ for which two of the branches with negative $c$ have the same character as the other two branches.

In the limit $k \rightarrow 0, m c^{2} \rightarrow 0,(2.11)$ reduces to

$$
\left(\beta_{1}-\beta_{0}\right) \gamma_{1}-\left(\alpha_{1}-\beta_{0}\right)^{2}=0
$$

which is the bifurcation condition for a (static) localized bulge to initiate from the uniform inflation solution (see FPL). We may note that the existence of four real solutions of (2.11) is guaranteed by the strong ellipticity (2.12) and $\left(\beta_{1}-\beta_{0}\right) \gamma_{1}-\left(\alpha_{1}-\beta_{0}\right)^{2}>0$. The latter two conditions are also sufficient for the stability of the uniformly inflated tube with respect to axi-symmetric perturbations (Chen 1997).

On the other hand, under the single assumption that the fluid is stationary prior to wave propagation, corresponding to $v_{\mathrm{f} \infty}=0$, the dispersion relation (2.8) reduces to

$$
\begin{gathered}
\left(k^{2} m^{2}+2 m\right) c^{4}-\left(m \alpha_{0} k^{2}+m \gamma_{1} k^{2}-m \beta_{0}+m \beta_{1}+2 \gamma_{1}\right) c^{2} \\
-\left(\alpha_{1}-\beta_{0}\right)^{2}+k^{2} \alpha_{0} \gamma_{1}-\beta_{0} \gamma_{1}+\beta_{1} \gamma_{1}=0 .
\end{gathered}
$$

For $k \ll 1$, we look for a series solution of the form

$$
c=\zeta\left(1-\sigma k^{2}\right)+O\left(k^{4}\right)
$$


where $\zeta$ and $\sigma$ are constants. On substituting (2.15) into (2.14) and equating the coefficients of $k^{0}$ and $k^{2}$ to zero, we find that $\zeta$ satisfies

$$
2 m \zeta^{4}+\left(m \beta_{0}-m \beta_{1}-2 \gamma_{1}\right) \zeta^{2}+\left(\beta_{1}-\beta_{0}\right) \gamma_{1}-\left(\alpha_{1}-\beta_{0}\right)^{2}=0
$$

and $\sigma$ is given by

$$
\sigma=\frac{m^{2} \zeta^{4}-\left(m \alpha_{0}+m \gamma_{1}\right) \zeta^{2}+\alpha_{0} \gamma_{1}}{2 \zeta^{2}\left(4 m \zeta^{2}+m \beta_{0}-m \beta_{1}-2 \gamma_{1}\right)} .
$$

Demiray and Dost (1998) derived a dispersion relation by treating the fluid flow as being two-dimensional and assuming that the flow is stationary prior to wave propagation. As expected, our leading order result (2.16) agrees with their result, but their expression for $\sigma$ takes the slightly different form

$$
\sigma=\frac{\left(m^{2}+m / 4\right) \zeta^{4}-\left(m \alpha_{0}+\gamma_{1} / 4+m \gamma_{1}\right) \zeta^{2}+\alpha_{0} \gamma_{1}}{2 \zeta^{2}\left(4 m \zeta^{2}+m \beta_{0}-m \beta_{1}-2 \gamma_{1}\right)}
$$

The extra terms in (2.18) are from dependence of fluid flow on the radial variable and can be seen to be negligible in the limit $m \gg 1$.

\section{$3 \quad$ Solitary-wave solutions}

For a travelling-wave solution in which the dependence on $Z$ and $t$ is through $Z-\hat{c} t$, EJ showed that (2.5) and (2.6) have two integrals (conservation laws). After some manipulation, we find that their original integrals can be rewritten in the simpler form

$$
\begin{gathered}
W\left(\lambda_{1}, \lambda_{2}\right)-\lambda_{2} W_{2}+\frac{1}{2} \rho \hat{c}^{2} \lambda_{2}^{2}=C_{1}, \\
\left(W_{2} / \lambda_{2}-\rho \hat{c}^{2}\right) z^{\prime}-\frac{1}{2} \lambda_{1}^{2}\left\{p_{\infty}+v_{\mathrm{f}}\left(1+\lambda_{1 \infty}^{4} / \lambda_{1}^{4}\right)\right\}=C_{2},
\end{gathered}
$$

where the two constants $C_{1}$ and $C_{2}$ each take the value of the corresponding left hand side evaluated at $\pm \infty$. Here and hereafter, dependent variables are all functions of $Z-\hat{c} t$, a prime now denotes differentiation with respect to $Z-\hat{c} t$, and we shall use $Z$ to denote $Z-\hat{c} t$ to avoid introducing extra notation.

The above conservation laws with $\hat{c}=0$ are well-known in the finite elasticity community: the conservation law (3.2) can be obtained by straightforward integration of (2.5); the other conservation law (3.1) was first noted by Pipkin (1968).

We observe that the two equations (3.1) and (3.2) may be rewritten as

$$
f\left(\lambda_{1}, \lambda_{2}\right)=0, \quad z^{\prime}=g\left(\lambda_{1}, \lambda_{2}\right)
$$

where

$$
f=W\left(\lambda_{1}, \lambda_{2}\right)-\lambda_{2} W_{2}+\frac{1}{2} \rho \hat{c}^{2} \lambda_{2}^{2}-C_{1}
$$




$$
g=\left(W_{2} / \lambda_{2}-\rho \hat{c}^{2}\right)^{-1}\left\{\frac{1}{2} \lambda_{1}^{2}\left\{p_{\infty}+v_{\mathrm{f}}\left(1+\lambda_{1 \infty}^{4} / \lambda_{1}^{4}\right)\right\}+C_{2}\right\} .
$$

Equations (3.3) are of the same form as those studied by FPL. We may thus follow these authors' methodology and derive the amplitude equations as follows. Firstly, we write

$$
\lambda_{1}=\lambda_{1 \infty}+w
$$

and assume $|w|$ to be small. Equation $(3.3)_{1}$ then defines $\lambda_{2}$ as a function of $w$ implicitly. This function can be expanded as

$$
\lambda_{2}=\lambda_{2 \infty}+d_{1} w+\frac{1}{2} d_{2} w^{2}+O\left(w^{3}\right)
$$

where the coefficients $d_{1}, d_{2}$ etc can be obtained by substituting (3.4) and (3.5) into the left hand side of $(3.3)_{1}$, expanding in terms of $w$, and then equating the coefficients of $w^{0}, w, w^{2}$, etc to zero. This can easily be carried out using a symbolic manipulation package such as Mathematica. Next, we substitute (3.4) and (3.5) into (3.3) $)_{2}$ and expand in terms of $w$ to obtain

$$
z^{\prime}=\lambda_{2 \infty}+g_{1} w+\frac{1}{2} g_{2} w^{2}+O\left(w^{3}\right),
$$

where $g_{1}$ and $g_{2}$, are constants with known expressions. Finally, on substituting (3.4)-(3.6) into $(2.2)_{1}$ and again expanding in terms of $w$, we arrive at the amplitude equation

$$
\left(w^{\prime}\right)^{2}=\omega\left(c, \lambda_{1 \infty}, \lambda_{2 \infty}\right) w^{2}+\gamma\left(c, \lambda_{1 \infty}, \lambda_{2 \infty}\right) w^{3}+O\left(w^{4}\right)
$$

where with $X$ denoting $m c^{2}$,

$$
\begin{gathered}
\omega\left(c, \lambda_{1 \infty}, \lambda_{2 \infty}\right)=\frac{\lambda_{2 \infty}^{2}}{\lambda_{1 \infty}^{2}} \cdot \frac{\left[2\left(v_{\mathrm{f} \infty}-c\right)^{2}+\beta_{0}-\beta_{1}\right]\left(X-\gamma_{1}\right)-\left(\alpha_{1}-\beta_{0}\right)^{2}}{\left(X-\alpha_{0}\right)\left(X-\gamma_{1}\right)}, \\
\gamma\left(c, \lambda_{1 \infty}, \lambda_{2 \infty}\right)=\frac{\lambda_{2 \infty}^{2}}{\lambda_{1 \infty}^{3}} \cdot \frac{\zeta_{4} X^{4}+\zeta_{3} X^{3}+\zeta_{2} X^{2}+\zeta_{1} X+\zeta_{0}}{3\left(X-\alpha_{0}\right)^{2}\left(\gamma_{1}-X\right)^{3}},
\end{gathered}
$$

and

$$
\begin{aligned}
\zeta_{4}= & 6\left(v_{\mathrm{f} \infty}-c\right)^{2}+2 \beta_{2}, \\
\zeta_{3}= & \left(v_{\mathrm{f} \infty}-c\right)^{2}\left(6 \beta_{0}-6 \alpha_{0}-12 \alpha_{1}-18 \gamma_{1}\right) \\
& +3 \beta_{0}^{2}+6 \alpha_{1} \alpha_{2}-6 \alpha_{1} \beta_{0}-6 \alpha_{2} \beta_{0}+3 \alpha_{1} \beta_{1}-2 \alpha_{0} \beta_{2}-6 \beta_{2} \gamma_{1}, \\
\zeta_{2}= & \left(v_{\mathrm{f} \infty}-c\right)^{2}\left(12 \alpha_{0} \alpha_{1}+24 \gamma_{1} \alpha_{1}+18 \gamma_{1}^{2}-12 \alpha_{0} \beta_{0}+18 \alpha_{0} \gamma_{1}-6 \beta_{0} \gamma_{1}\right) \\
+ & 3 \alpha_{1}^{3}-6 \beta_{0} \alpha_{1}^{2}+6 \gamma_{2} \alpha_{1}^{2}+3 \beta_{0}^{2} \alpha_{1}-6 \alpha_{0} \alpha_{2} \alpha_{1}+6 \alpha_{0} \beta_{0} \alpha_{1} \\
& -3 \alpha_{0} \beta_{1} \alpha_{1}-12 \alpha_{2} \gamma_{1} \alpha_{1}+12 \beta_{0} \gamma_{1} \alpha_{1}-6 \beta_{1} \gamma_{1} \alpha_{1}-12 \beta_{0} \gamma_{2} \alpha_{1} \\
& -6 \alpha_{0} \beta_{0}^{2}+6 \beta_{2} \gamma_{1}^{2}+6 \alpha_{0} \alpha_{2} \beta_{0}+3 \alpha_{0} \beta_{0} \beta_{1}-3 \beta_{0}^{2} \gamma_{1} \\
+ & 12 \alpha_{2} \beta_{0} \gamma_{1}-3 \beta_{0} \beta_{1} \gamma_{1}+6 \alpha_{0} \beta_{2} \gamma_{1}+6 \beta_{0}^{2} \gamma_{2},
\end{aligned}
$$




$$
\begin{aligned}
\zeta_{1}= & \left(v_{\mathrm{f} \infty}-c\right)^{2}\left(24 \alpha_{0} \beta_{0} \gamma_{1}-12 \gamma_{1}^{2} \alpha_{1}-24 \alpha_{0} \gamma_{1} \alpha_{1}-6 \gamma_{1}^{3}-18 \alpha_{0} \gamma_{1}^{2}-6 \beta_{0} \gamma_{1}^{2}\right) \\
& -3 \alpha_{0} \alpha_{1}^{3}-3 \gamma_{1} \alpha_{1}^{3}+2 \gamma_{3} \alpha_{1}^{3}+9 \alpha_{0} \beta_{0} \alpha_{1}^{2}+3 \beta_{0} \gamma_{1} \alpha_{1}^{2}-6 \alpha_{0} \gamma_{2} \alpha_{1}^{2} \\
& -6 \gamma_{1} \gamma_{2} \alpha_{1}^{2}-6 \beta_{0} \gamma_{3} \alpha_{1}^{2}-9 \alpha_{0} \beta_{0}^{2} \alpha_{1}+6 \alpha_{2} \gamma_{1}^{2} \alpha_{1}-6 \beta_{0} \gamma_{1}^{2} \alpha_{1}+3 \beta_{1} \gamma_{1}^{2} \alpha_{1} \\
& +3 \beta_{0}^{2} \gamma_{1} \alpha_{1}+12 \alpha_{0} \alpha_{2} \gamma_{1} \alpha_{1}-12 \alpha_{0} \beta_{0} \gamma_{1} \alpha_{1}+6 \alpha_{0} \beta_{1} \gamma_{1} \alpha_{1} \\
& +12 \alpha_{0} \beta_{0} \gamma_{2} \alpha_{1}+12 \beta_{0} \gamma_{1} \gamma_{2} \alpha_{1}+6 \beta_{0}^{2} \gamma_{3} \alpha_{1}+3 \alpha_{0} \beta_{0}^{3}-2 \beta_{2} \gamma_{1}^{3} \\
& -3 \beta_{0}^{2} \gamma_{1}^{2}-6 \alpha_{2} \beta_{0} \gamma_{1}^{2}+6 \beta_{0} \beta_{1} \gamma_{1}^{2}-6 \alpha_{0} \beta_{2} \gamma_{1}^{2}-3 \beta_{0}^{3} \gamma_{1}+12 \alpha_{0} \beta_{0}^{2} \gamma_{1} \\
& -12 \alpha_{0} \alpha_{2} \beta_{0} \gamma_{1}-6 \alpha_{0} \beta_{0} \beta_{1} \gamma_{1}-6 \alpha_{0} \beta_{0}^{2} \gamma_{2}-6 \beta_{0}^{2} \gamma_{1} \gamma_{2}-2 \beta_{0}^{3} \gamma_{3}, \\
\zeta_{0}=\quad & \left.v_{\mathrm{f} \infty}-c\right)^{2}\left(6 \alpha_{0} \gamma_{1}^{3}+6 \beta_{0} \gamma_{1}^{3}+12 \alpha_{0} \alpha_{1} \gamma_{1}^{2}-12 \alpha_{0} \beta_{0} \gamma_{1}^{2}\right)-6 \alpha_{0} \beta_{0}^{2} \gamma_{1}\left(\gamma_{1}-\gamma_{2}\right) \\
+ & \left(2 \gamma_{3}-3 \gamma_{1}\right)\left(\alpha_{0} \beta_{0}^{3}-\alpha_{0} \alpha_{1}^{3}+3 \alpha_{0} \alpha_{1}^{2} \beta_{0}-3 \alpha_{0} \beta_{0}^{2} \alpha_{1}\right) \\
+ & 3 \beta_{0} \gamma_{1}^{2} \alpha_{1}^{2}+6 \alpha_{0} \alpha_{1}^{2} \gamma_{1} \gamma_{2}-6 \beta_{0}^{2} \gamma_{1}^{2} \alpha_{1}-6 \alpha_{0} \alpha_{2} \gamma_{1}^{2} \alpha_{1}-3 \alpha_{0} \beta_{1} \gamma_{1}^{2} \alpha_{1} \\
+ & 6 \alpha_{0} \beta_{0} \gamma_{1} \alpha_{1}\left(\gamma_{1}-2 \gamma_{2}\right)+3 \beta_{0}^{2} \gamma_{1}^{3}-3 \beta_{0} \beta_{1} \gamma_{1}^{3}+2 \alpha_{0} \beta_{2} \gamma_{1}^{3} \\
+ & 3 \beta_{0}^{3} \gamma_{1}^{2}+6 \alpha_{0} \alpha_{2} \beta_{0} \gamma_{1}^{2}+3 \alpha_{0} \beta_{0} \beta_{1} \gamma_{1}^{2} .
\end{aligned}
$$

In the limit $c \rightarrow 0, v_{\mathrm{f} \infty} \rightarrow 0$, (3.7) reduces to the amplitude equation given by FPL. For each fixed choice of $\lambda_{1 \infty}$ and $\lambda_{2 \infty}$, the travelling wave problem can be viewed as a bifurcation problem with the speed $c$ acting as the bifurcation parameter. The bifurcation condition is given by

$$
\omega\left(c, \lambda_{1 \infty}, \lambda_{2 \infty}\right)=0,
$$

which, as expected, is equivalent to the dispersion relation (2.8) for $k=0$.

Denote by $c_{1}$ a solution of (3.8), write $\varepsilon=\left|c-c_{1}\right|$, and assume $\varepsilon$ to be small. Equation (3.7) may be replaced by

$$
\left(w^{\prime}\right)^{2}=\omega_{c}\left(c_{1}, \lambda_{1 \infty}, \lambda_{2 \infty}\right)\left(c-c_{1}\right) w^{2}+\gamma\left(c_{1}, \lambda_{1 \infty}, \lambda_{2 \infty}\right) w^{3}+O\left(\varepsilon^{2} w^{2}, w^{4}\right)
$$

where $\omega_{c}$ denotes $\partial \omega / \partial c$. Requiring the first three terms in (3.9) to have the same order, we deduce that $w$ must necessarily be of order $\varepsilon$ and its variation takes place on a lengthscale of order $\varepsilon^{-1 / 2}$. Thus, in terms of the new variables $\xi$ and $a$ defined by

$$
w=-\frac{2 \varepsilon\left|\omega_{c}\right|}{3 \gamma} a(\xi), \quad \xi=\sqrt{\varepsilon\left|\omega_{c}\right|} Z,
$$

equation (3.9) takes the form

$$
\left(a^{\prime}\right)^{2}=\operatorname{sgn}\left[\left(c-c_{1}\right) \omega_{c}\right] a^{2}-\frac{2}{3} a^{3}+O(\varepsilon),
$$

where we have suppressed the dependence of $\omega_{c}$ and $\gamma$ on $c_{1}, \lambda_{1 \infty}$ and $\lambda_{2 \infty}$. It can easily be shown that provided $\left(c-c_{1}\right) \omega_{c}>0$, this equation, with the $O(\varepsilon)$ terms neglected, has a solitary-wave solution given by $a=a_{0}$, where

$$
a_{0}=\frac{3}{2} \operatorname{sech}^{2} \frac{\xi}{2} \text {. }
$$


We observe that when $\gamma<0$ the solitary wave is a wave of elevation $(w>0)$, whereas when $\gamma>0$ the solitary wave is a wave of depression $(w<0)$. The sign of $\omega_{c}$ determines whether the solitary wave is supersonic (i.e. $c>c_{1}$ ) or subsonic (i.e. $c<c_{1}$ ).

EJ also showed that a differential equation of the form $\left(w^{\prime}\right)^{2}=f_{c}(w)$ can be derived from the two conservation laws (3.1) and (3.2), where the function $f_{c}(w)$ on the right hand side can, of course, only be determined numerically. They further deduced that this exact equation has a solitary-wave solution only if $f_{c}(0)=0, f_{c}^{\prime}(0)=0, f_{c}^{\prime \prime}(0)>0$ and $f_{c}\left(w_{\max }\right)=0$ for some positive $w_{\max }$. They did not, however, seem to realize that with an appropriate choice of $c$ and rescaling of $w$ these conditions are always satisfied, whether the fluid is initially stationary or not, if $c$ is close to a linear wave speed because then $w$ is small and $f_{c}(w)$ has the expansion given by (3.9).

We would have liked to compare the differentiated form of our equation (3.9) with Demiray and Dost's (1998) equation (46), but it seems that their series expansion (19) is not self-consistent: $\gamma_{1}(\partial w / \partial z)^{2}+\left(\gamma_{1} / 2\right)(\partial u / \partial z)^{2}$ should be added to the first expression and $\alpha_{1}(\partial w / \partial z)^{2}+\left(\alpha_{1} / 2\right)(\partial u / \partial z)^{2}$ should be added to the second expression in their equation (19). We thus decide not to make any comparisons.

\section{The role of axial displacement}

A very simple model that has been adopted in some previous studies is one in which $u$ and $w^{\prime 2}$ are viewed to be negligible in a small-amplitude and long-wave approximation; see, for instance, Demiray (1996) and Epstein and Johnston (1999). In this case, we have

$$
r=\lambda_{1}=\lambda_{1 \infty}+w, \quad \lambda_{2} \equiv \lambda_{2 \infty}
$$

The first term in (2.6) is approximated by

$$
\left[\sigma_{2} \frac{r^{\prime}}{\lambda_{2}^{2}}\right]^{\prime}=\frac{1}{\lambda_{2 \infty}^{2}}\left[\sigma_{2} w^{\prime}\right]^{\prime}=\frac{1}{\lambda_{2 \infty}^{2}}\left(\sigma_{2} w^{\prime \prime}+\frac{\partial \sigma_{2}}{\partial w} w^{\prime 2}\right) \approx \frac{\sigma_{2}}{\lambda_{2 \infty}^{2}} w^{\prime \prime} .
$$

Equation (2.6) is then approximated by

$$
\left(\frac{\sigma_{2}}{\lambda_{2 \infty}^{2}}-\rho \hat{c}^{2}\right) w^{\prime \prime}-\frac{\sigma_{1}}{\lambda_{1}}+p \lambda_{1} \lambda_{2 \infty}=0
$$

Linearizing this equation in terms of $w$ and taking $\hat{v}_{\mathrm{f} \infty}=0$, we obtain

$$
\left(\alpha_{0}-m c^{2}\right) w^{\prime \prime}+\lambda_{1 \infty}^{-2} \lambda_{2 \infty}^{2}\left(2 c^{2}+\beta_{0}-\beta_{1}\right) w=0
$$

where the various constants are given by (2.9) and (2.10). Thus, taking $w$ to be proportional to $\mathrm{e}^{\mathrm{i} \hat{k}(Z-\hat{c} t)}$, we obtain the dispersion relation

$$
k^{2}\left(m c^{2}-\alpha_{0}\right)+2 c^{2}+\beta_{0}-\beta_{1}=0,
$$


where $k$ is given by $(2.9)_{5}$. The only way to justify this dispersion relation is to take the limit $m \gg 1, k \ll 1$ with $m k^{2}=O(1)$ in (2.14). In this limit, (2.14) can be replaced by

$$
\left(k^{2} m+2\right) c^{2}+\beta_{0}-\beta_{1}=0,
$$

which agrees with (4.4) since the term $k^{2} \alpha_{0}$ in (4.4) is negligible. However, when $m k^{2}=O(1)$, we cannot obtain a series expansion for $c$ in terms of $k$ from (4.5), but such an expansion is essential in the small-amplitude and long-wavelength approximation. In fact, it was assumed in some previous studies that

$$
c=\sqrt{\left(\beta_{1}-\beta_{0}\right) / 2}+O(k)
$$

We note that the correction term $\sigma k^{2}$ in (2.15) becomes $O(1)$ and the expansion (2.15) breaks down as well. Thus, we conclude that it is not asymptotically consistent to ignore the axial displacement.

The nonlinear equation in this approximation is given by

$$
\begin{gathered}
w^{\prime \prime}=\frac{\lambda_{2 \infty}^{2}\left(2 c^{2}+\beta_{0}-\beta_{1}\right)}{\lambda_{1 \infty}^{2}\left(m c^{2}-\alpha_{0}\right)} w \\
-\frac{\lambda_{2 \infty}^{2}\left(m\left(3 c^{2}+\beta_{2}\right) c^{2}+\alpha_{1}\left(-2 c^{2}-\beta_{0}+\beta_{1}\right)-\alpha_{0}\left(3 c^{2}+\beta_{2}\right)\right)}{\lambda_{1 \infty}^{3}\left(m c^{2}-\alpha_{0}\right)^{2}} w^{2}+O\left(w^{3}\right) .
\end{gathered}
$$

Denoting $\sqrt{\left(\beta_{1}-\beta_{0}\right) / 2}$ by $\hat{c}_{0}$, expanding the right hand side of (4.6) in a small neighborhood of $c=\hat{c}_{0}$, and then neglecting terms of order $\left(c-\hat{c}_{0}\right)^{2} w$ or $w^{3}$, we obtain

$$
w^{\prime \prime}=-\frac{8 \hat{c}_{0} \lambda_{2 \infty}^{2}}{\lambda_{1 \infty}^{2}\left(2 \alpha_{0}+m\left(\beta_{0}-\beta_{1}\right)\right)}\left(c-\hat{c}_{0}\right) w-\frac{\lambda_{1 \infty}^{2}\left(3 \beta_{0}-3 \beta_{1}-2 \beta_{2}\right)}{\lambda_{1 \infty}^{3}\left(2 \alpha_{0}+m\left(\beta_{0}-\beta_{1}\right)\right)} w^{2} .
$$

This reproduces Demiray's (1996) equation (34) which was derived using a multiple-scale expansion.

\section{Persistence of the solitary wave solutions}

From now on we assume that $\left(c-c_{1}\right) \omega_{c}>0$. On differentiating (3.10) with respect to $\xi$, we obtain

$$
\mathcal{M}(a) \equiv a^{\prime \prime}-a+a^{2}+e(\varepsilon, a)=0,
$$

where $e(\varepsilon, a)=O(\varepsilon)$. Equation (5.1) is obviously reversible, that is, it is invariant under the action $\xi \rightarrow-\xi$. This reversibility means that we may restrict ourselves to the case when $a$ is an even function. We have the following result:

Lemma (Iooss \& Kirchgässner 1992): For a small enough $\varepsilon_{0}$ and $\varepsilon \in\left(0, \varepsilon_{0}\right]$ there exists a family of solitary wave solutions $a$ satisfying equation (5.1). Moreover,

$$
\left|a-a_{0}\right| \leq C \varepsilon \exp (-\tau|\xi|),
$$


where $C, \tau$ are constants, and $C>0,0<\tau<1$.

Proof. We first define the Banach functional spaces

$$
C_{\tau, j}^{e}=\left\{f_{0} \in C^{j}(\mathbb{R}): \sup _{\xi} \mid \exp (\tau|\xi|) f_{0}^{(m)}<\infty, \quad j=0,1,2 ; \quad m \leq j ; \quad f_{0}(\xi)=f_{0}(-\xi)\right\} .
$$

It is evident that $a_{0} \in C_{1,2}^{e}$.

Let $\mathcal{M}: C_{\tau, 2}^{e} \rightarrow C_{\tau, 0}^{e}$, where $\tau<1$. From the implicit function theorem the existence and uniqueness of $a \in C_{\tau, 2}^{e}, \varepsilon \in\left(0, \varepsilon_{0}\right]$ that satisfies the equation (5.1) will follow, if the operator

$$
\mathcal{L}=\left.\frac{\partial \mathcal{M}}{\partial a}\left(a_{0}\right)\right|_{\varepsilon=0}: C_{\tau, 2}^{e} \rightarrow C_{\tau, 0}^{e}
$$

has a bounded inverse. The proof of the first part of the assertion of the lemma is, therefore, reduced to the demonstration of this fact.

It can be easily seen that

$$
\mathcal{L}=\frac{\mathrm{d}^{2}}{\mathrm{~d} \xi^{2}}-1+2 a_{0}
$$

and the inverse exists if and only if the equation

$$
\mathcal{L} v=r
$$

has a unique solution for any $r \in C_{\tau, 0}^{e}$. The solvability of (5.2) follows from the following.

The homogeneous equation $\mathcal{L} v=0$ has no solutions in $C_{\tau, 2}^{e}$. In fact, it has one solution given by $v_{1}=a_{0}^{\prime}$ which is an odd function. Another linearly independent solution is

$$
v_{2}=-v_{1} \int^{\xi} v_{1}^{-2}(s) \mathrm{d} s=\frac{1}{24} \operatorname{sech}^{2} \frac{\xi}{2} \tanh \frac{\xi}{2}\left(30 \xi-32 \operatorname{coth} \frac{\xi}{2}+16 \sinh \xi+\sinh 2 \xi\right),
$$

which is an even function but grows like $\mathrm{e}^{\xi}$ as $\xi \rightarrow \infty$. It follows that the solution of (5.2) is unique if it exists.

The required solution for the inhomogeneous differential equation (5.2) can be obtained with the aid of the method of variation of parameters, and is given by the formula

$$
v=v_{2}(\xi) \int_{\xi}^{\infty} v_{1}(s) r(s) \mathrm{d} s+v_{1}(\xi) \int_{0}^{\xi} v_{2}(s) r(s) \mathrm{d} s .
$$

Moreover, it follows from (5.2) that $\|v\|_{C_{\tau, 2}^{e}} \leq\left\|\mathcal{L}^{-1}\right\| \cdot\|r\|_{C_{\tau, 0}^{e}} \leq C\|r\|_{C_{\tau, 0}^{e}}$, where $C>0$ is a constant.

The first part of the assertion of the Lemma is, therefore, proved. We next write the solution of (5.1) in the form $a=a_{0}+a_{1}$. The function $a_{1}$ obeys the equation

$$
\mathcal{L} a_{1}=h\left(\varepsilon, a_{1}\right),
$$

where $h(\cdot)=O\left(\varepsilon, \varepsilon\left|a_{1}\right|\right)$. From the boundedness of $\mathcal{L}$ and the implicit function theorem it then follows that there exists $\varepsilon_{0}>0$ such that for $\varepsilon \in\left[0, \varepsilon_{0}\right)$,

$$
a_{1}=O(\varepsilon) \in C_{\tau, 2}^{e},
$$



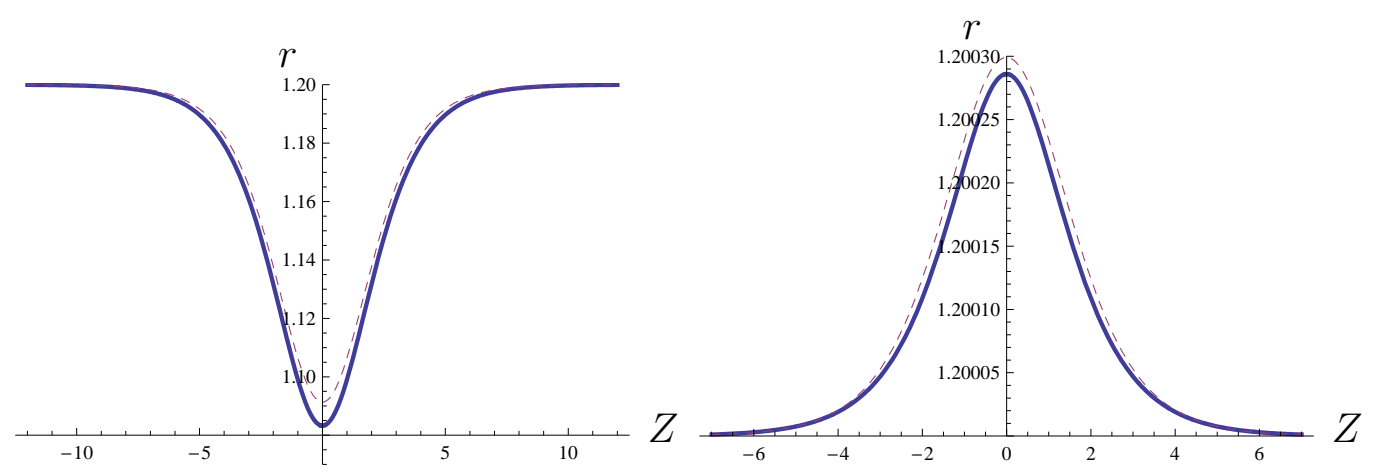

Figure 1: Solitary waves corresponding to $c^{2}=7$ (left plot) and $c^{2}=265$ (right plot), respectively. The solid line is the exact solution and the dashed line is the asymptotic solution given by (3.10).

from which the assertion of the Lemma concerning the norm follows immediately.

We have thus proved that the family of solitary wave solutions $a$ exists and is unique for small enough amplitudes. Returning to the unscaled variables, we conclude that the fluid-filled membrane tube supports a unique family of solitary wave solutions bifurcating from the trivial solution at each linear wave speed. The exact solitary-wave solutions can be obtained numerically using the method explained by EJ or FPL. As an example, we follow Demiray (1996) and EJ and consider the case when the strain-energy function is given by

$$
W=\frac{\mu}{2 \alpha}\left\{\exp \left[\alpha\left(\lambda_{1}^{2}+\lambda_{2}^{2}+\frac{1}{\lambda_{1}^{2} \lambda_{2}^{2}}-3\right)\right]-1\right\},
$$

where $\mu$ and $\alpha$ are material constants. We take

$$
\alpha=1.948, \quad \lambda_{1 \infty}=1.2, \quad \lambda_{2 \infty}=1.5, \quad m=0.5, \quad v_{\mathrm{f} \infty}=0 .
$$

In this case the scaled wave speed $c$ appears in the problem through $c^{2}$ and the bifurcation condition $\omega\left(c, \lambda_{1 \infty}, \lambda_{2 \infty}\right)=0$ has four roots $\pm c_{1}, \pm c_{2}$ where $c_{1}^{2}=8.6384, c_{2}^{2}=263.0176$. It is found that

$$
\begin{gathered}
\omega_{c}\left(c_{1}, \lambda_{1 \infty}, \lambda_{2 \infty}\right)=-2.0910, \quad \gamma\left(c_{1}, \lambda_{1 \infty}, \lambda_{2 \infty}\right)=5.6464, \\
\omega_{c}\left(c_{2}, \lambda_{1 \infty}, \lambda_{2 \infty}\right)=16.6598, \quad \gamma\left(c_{2}, \lambda_{1 \infty}, \lambda_{2 \infty}\right)=-3398.3050 .
\end{gathered}
$$

Thus, in accordance with the statement below (5.1), the two solitary waves that bifurcate from $c=c_{1}$ are subsonic and are waves of depression, whereas the two solitary waves that bifurcate from $c=c_{2}$ are supersonic and are waves of elevation. Figure 1 shows two such solitary wave profiles, corresponding to $c^{2}=7$ and $c^{2}=265$, respectively. In each case the exact solution is obtained by integrating three first-order ODEs for $\lambda_{1}(Z), \lambda_{2}(Z)$ and $\phi(Z)$ from $Z=0$ towards $\infty$, where $\phi$ is the angle between the meridian and the $Z$-axis (so that $\left.\cos \phi=z^{\prime} / \lambda_{2}, \sin \phi=r^{\prime} / \lambda_{2}\right)$. The initial conditions at $Z=0$ are obtained by solving (3.1) and (3.2) evaluated at $Z=0$, and are given by

$$
\lambda_{1}(0)=1.08327596, \quad \lambda_{2}(0)=1.56168283, \quad \phi(0)=0
$$


when $c^{2}=7$, and by

$$
\lambda_{1}(0)=1.20028598, \quad \lambda_{2}(0)=1.50335606, \quad \phi(0)=0
$$

when $c^{2}=265$. The excellent agreement between the asymptotic and exact results is of course simply a manifestation of our persistence result.

\section{Acknowledgements}

This research was supported by an INTAS Joint Project Grant (Ref. Nr 05-1000008-7921). The second author's contribution was also partly supported by Russian Foundation for Basic Research (Grant No. 08-01-00618).

\section{References}

1. Antar, N \& Demiray, H. (1999) Weakly nonlinear waves in a prestressed elastic tube containing a viscous fluid. Int. J. Engn Sci. 37, 1859-1876.

2. Budiansky, B. (1968) Notes on nonlinear shell theory. J. Appl. Mech. 35, 393-401.

3. Chen, Y-C. (1997) Stability and bifurcation of finite deformations of elastic cylindrical membranes - part I. stability analysis. Int. J. Solids Struct. 34, 1735-1749.

4. Cowley, S.J. (1983) On the wavetrains associated with elastic jumps on fluid-filled elastic tubes. Q.J. Mech. Appl. Maths 36, 289-312.

5. Davies, P.J. (1991) A simple derivation of necessary and sufficient conditions for the strong ellipticity of isotropic hyperelastic materials in plane strain. J. Elast. 26, 291-296.

6. Demiray, H. (1996) Solitary waves in initially stressed thin elastic tubes. Int. J. Non-Linear Mech., 32, 1165-1176.

7. Demiray, H. (1997) Solitary waves in prestressed elastic tubes. Bull. Math. Biol., 58, 939-955.

8. Demiray, H. \& Dost, S. (1998) Axial and transverse solitary waves in prestressed elastic tubes. ARI 50, 1065-1079.

9. Epstein, M \& Johnston, C. (1999) Improved solution for solitary waves in arteries. J. Math. Biol. 39, 1-18.

10. Epstein, M \& Johnston, C. (2001) On the exact speed and amplitude of solitary waves in fluid-filled elastic tubes. Proc. R. Soc. Lond. A 457, 1195-1213. 
11. Erbay, S., Erbay, H. \& Dost, S. (1992 ) Wave propagation in fluid-filled nonlinear viscoelastic tubes. Acta Mech. 95, 87-102.

12. Fu, Y. B., Pearce, S. P. \& Liu, K. K. (2008) Post-bifurcation analysis of a thin-walled hyperelastic tube under inflation. Int. J. Non-Linear Mech. 43, 697-706.

13. Hashizume, Y. (1985) Nonlinear pressure waves in a fluid-filled elastic tube. J. Phys Soc. Jpn 54, 3305-3312.

14. Iooss, G. \& Kirchgässner, K. (1992) Water waves for small surface tension: an approach via normal form. Proc. Roy. Soc. Edin. A 122, 267-299.

15. Johnson, R.S. (1971) Nonlinear waves in fluid filled elastic tubes and related phenomenon. Ph.D. dissertation, University of London.

16. Ogden, R.W. (1997) Non-linear elastic deformations. Dover, New York.

17. Pipkin, A.C. (1968) Integration of an equation in membranes theory. ZAMP, 19, 818-819.

18. Tait, R.J., Bryant, T. \& Haddow, J.B. (1981) Wave propagation in a fluid-filled elastic tube. Acta Mech. 38, 71-83.

19. Yomosa, Y. (1987) Solitary waves in large blood vessels. J. Phys Soc. Jpn 56, 506-520. 